Acta Crystallographica Section B

Structural

Science

ISSN 0108-7681

H. D. Flack, ${ }^{a *}$ G. Bernardinelli, ${ }^{a}$ D. A. Clemente, ${ }^{b}$ A. Linden ${ }^{c}$ and A. L. Spek

aLaboratoire de Cristallographie, University of Geneva, Switzerland, 'b Dipartimento dei Materiali e delle Risorse Naturali, Università di Trieste sede di Pordenone, Italy, 'Institute of Organic Chemistry, University of Zurich, Switzerland, and ${ }^{\mathbf{d}}$ Department of Crystal and Structural Chemistry, Bijvoet Center for Biomolecular Research, Utrecht University, The Netherlands

Correspondence e-mail:

howard.flack@cryst.unige.ch

\title{
Centrosymmetric and pseudo-centrosymmetric structures refined as non-centrosymmetric
}

The behaviour of the Flack parameter for centrosymmetric and pseudo-centrosymmetric crystal structures based on crystal structures published as being non-centrosymmetric is presented. It is confirmed for centrosymmetric structures that the value obtained for the Flack parameter is critically dependent on the Friedel coverage of the intensity data, approaching 0.5 for a coverage of $100 \%$ and sticking near the starting value for a coverage of $0 \%$. For pseudo-centrosymmetric structures, even those very close to being centrosymmetric, it is found that it is often possible to obtain significant values of the Flack parameter. A theoretical basis for this surprising result is established. It has also been possible to establish an a priori estimate of the standard uncertainty of the Flack parameter based only on the chemical composition of the compound and the wavelength of the radiation. The paper concludes with preliminary presentations of bias in the Flack parameter and of inconsistent chemical and crystallographic data.

\section{Introduction}

Flack \& Bernardinelli (2006) have recently published a short analysis of values of the Flack (1983) parameter obtained from 23 centrosymmetric structures but published in Inorganica Chimica Acta as being non-centrosymmetric. This analysis resides on the identification of the appropriate centrosymmetric space groups of these structures by Clemente (2005). In the present paper we extend our study of the values of the Flack parameter to a far greater number of crystal structures, published as being non-centrosymmetric but having subsequently been identified in published and unpublished works as being centrosymmetric.

It has also been possible to investigate the behaviour of the Flack parameter for pseudo-centrosymmetric structures. Although there are no published lists of such structures, it has nevertheless been possible to identify a sufficiently large number from our own and other sources to make a statistically significant study.

Finally we undertake a short description and discussion of a new method for predicting a priori the standard uncertainty of the Flack (1983) parameter based on the chemical composition of the compound under study, of bias in the Flack (1983) parameter, and of troublesome reported cases of incompatibility between chemical and crystallographic evidence.

\section{Centrosymmetric structures treated as non-centrosymmetric}

From the publications of Clemente $(2005,2006)$, Clemente \& Marzotto (2003, 2004), Herbstein et al. (2002), Herbstein \&
Received 2 May 2006

Accepted 8 June 2006
(C) 2006 International Union of Crystallography Printed in Great Britain - all rights reserved 
Table 1

Values of the Flack parameter, arranged in ascending order of value by columns, for 28 crystal-structure reports based on a Friedel coverage of $100 \%$.

Values of $x$ reported larger than 0.5 have been transformed into $1.0-x$ in this table.

\begin{tabular}{lllll}
\hline $0.15(4)$ & $0.30(13)$ & $0.416(13)$ & $0.46(12)$ & $0.481(14)$ \\
$0.23(8)$ & $0.30(3)$ & $0.42(5)$ & $0.46(6)$ & $0.487(13)$ \\
$0.23(8)$ & $0.3(1)$ & $0.44(10)$ & $0.46(6)$ & $0.49(2)$ \\
$0.28(16)$ & $0.31(2)$ & $0.44(4)$ & $0.47(2)$ & $0.50(3)$ \\
$0.29(10)$ & $0.31(8)$ & $0.45(3)$ & $0.48(5)$ & \\
$0.30(2)$ & $0.38(4)$ & $0.452(19)$ & $0.48(2)$ & \\
\hline
\end{tabular}

Marsh (1998), Marsh (1999, 2002, 2004, 2005), Marsh et al. (2002) and Marsh \& Spek (2001), crystal structures were extracted corresponding to the following three criteria: (i) at least one atom should be of an element heavier than Ar, (ii) the paper should be published after 1995; and (iii) a centre of symmetry needs adding to the space group. Criterion (i) was chosen to try to ensure small standard uncertainties on the Flack parameters determined and hence significant values of the parameter itself. Criterion (ii) was chosen to correspond to the widespread use of the Flack (1983) parameter in published crystal-structure determinations. Criterion (iii) allows us to study crystal structures which were published as being noncentrosymmetric but after careful examination by the authors cited above were shown to be centrosymmetric. This reassignment of space group is based on a search, often automated, in the set of atomic coordinates of the determined structure for supplementary symmetries which are not part of the chosen space group. Often deviations of interatomic distances and angles between chemically equivalent bonds in the same or equivalent molecules can be ascribed to instabilities in the least-squares refinement. Aberrant shapes in the ellipsoid representation of atomic displacement parameters also provide an indication of an incorrect choice of space group. Frequently it is not possible to carry out a leastsquares refinement in the higher symmetry space group due to the diffraction intensity data being unavailable as supplementary material to the published structure. As sources of data, the published papers and any supplementary material freely available for download from the journal's online site, the CIF archive of the Cambridge Crystallographic Data Centre (CCDC) or sometimes from the Fachsinformationszentrum (FIZ), Karlsruhe, were used. Authors were never contacted for supplementary material. Each paper available in searchable digital form was scanned for the following strings of characters: BASF, Flack, absolute, enantio, $\mathrm{CD}$, optical, chiral, racem. It is of particular importance in the current study to determine whether pairs of Friedel opposites $h k l$ and $-h-k-l$ (or symmetry equivalent) have been measured and used separately in the least-squares refinement. For this purpose we define the Friedel coverage as a measure of the completeness of the diffraction intensity data with regard to inversion in the origin of reciprocal space. If for each value of $h k l$ the intensity of the Friedel opposite $-h-k-l$ (or one symmetry-equivalent to it) has not been measured, then the Friedel coverage is $0 \%$. However, if for each value of
Table 2

Values of the Flack parameter, arranged in ascending order of value by columns, for 67 crystal-structure reports based on a Friedel coverage of $0 \%$.

\begin{tabular}{lllll}
\hline$-0.79(14)$ & $-0.04(4)$ & $0.03(4)$ & $0.05(3)$ & $0.09(3)$ \\
$-0.26(13)$ & $-0.03(2)$ & $0.03(3)$ & $0.059(11)$ & $0.09(2)$ \\
$-0.18(8)$ & $-0.026(2)$ & $0.03(2)$ & $0.06(8)$ & $0.11(8)$ \\
$-0.14(9)$ & $-0.01(5)$ & $0.03(4)$ & $0.06(3)$ & $0.11(7)$ \\
$-0.14(5)$ & $-0.01(3)$ & $0.037(12)$ & $0.06(4)$ & $0.13(10)$ \\
$-0.11(12)$ & $-0.01(3)$ & $0.037(9)$ & $0.06(3)$ & $0.13(8)$ \\
$-0.09(9)$ & $0.00(7)$ & $0.04(7)$ & $0.068(10)$ & $0.19(10)$ \\
$-0.07(4)$ & $0.0(1)$ & $0.04(11)$ & $0.07(6)$ & $0.31(9)$ \\
$-0.07(4)$ & $0.00(3)$ & $0.04(4)$ & $0.07(8)$ & $0.31(16)$ \\
$-0.05(4)$ & $0.000(14)$ & $0.04(3)$ & $0.08(6)$ & 0.57 \\
$-0.04(5)$ & $0.01(4)$ & $0.04(5)$ & $0.08(7)$ & $0.67(4)$ \\
$-0.04(8)$ & $0.01(5)$ & $0.04(3)$ & $0.08(4)$ & \\
$-0.04(7)$ & $0.01(6)$ & $0.047(13)$ & $0.08(7)$ & \\
$-0.04(5)$ & $0.03(2)$ & $0.05(16)$ & $0.09(4)$ & \\
\hline
\end{tabular}

$h k l$, both the reflection $h k l$ and its Friedel opposite $-h-k-l$ (or one symmetry-equivalent to it) have been measured and used separately in the least-squares refinement, then the Friedel coverage is $100 \%$. For some special values of the indices $h k l$ (e.g. $h 0 l$ in space group $P 2_{1}$ ) the reflections $h k l$ and $-h-k-l$ are symmetry equivalent and are generally called centrosymmetric reflections. The centrosymmetric reflections are not included in the calculation of the Friedel coverage. From the information provided in the original paper and supplementary publication it is not always possible to determine unequivocally the Friedel coverage.

A table containing the pertinent aspects of the set of 231 centrosymmetric crystal-structure analyses refined as noncentrosymmetric is presented in the supplementary material. ${ }^{\mathbf{1}}$ As concerns the reporting of the absolute-structure determination, 89 of these have no report and eight of them have a dismissive textual report. 24 of them report ' $x=0.00$ ' for the absolute-structure determination, implying that due to some data manipulation error (Clegg, 2003) the refinement of the Flack parameter was not reported or not carried out. Thus, overall $52 \%$ of the structure reports are inadequate in their reporting of the absolute-structure determination. In five of the remaining crystal-structure analyses the standard uncertainty on the Flack parameter is too high for the value itself to be interpretable. For the remaining crystal-structure determinations, 28 analyses have been carried out with a Friedel coverage of $100 \%, 67$ with a coverage of $0 \%$ and 10 with an intermediate coverage.

Table 1 gives values of the Flack parameter reported in the 28 structure analyses based on a Friedel coverage of $100 \%$. One can see that the Flack parameters tend to take a value of 0.5 although the spread is considerable. With a Friedel coverage of $100 \%$, both observed intensities $I_{\text {obs }}(h k l)$ and $I_{\text {obs }}(-h-k-l)$ are present in the least-squares refinement, and in the absence of random and systematic effects these would have the same intensity since the structure is centrosymmetric. In the non-centrosymmetric refinement, equality

\footnotetext{
${ }^{\mathbf{1}}$ Supplementary data for this paper are available from the IUCr electronic archives (Reference: GP5012). Services for accessing these data are described at the back of the journal.
} 
of the model intensities $I_{\text {model }}(h k l)$ and $I_{\text {model }}(-h-k-l)$ is achieved when the Flack parameter takes a value of 0.5. Other details of this argument are given by Flack \& Bernardinelli (2006), and Table 1 confirms this analysis.

Table 2 gives values of the Flack parameter reported in the 67 structure analyses based on a Friedel coverage of $0 \%$. The values are arranged around the value of zero but with a tendency to be positive. With a Friedel coverage of $0 \%$ only the observed intensities $I_{\mathrm{obs}}(h k l)$ are present in the refinement and $I_{\mathrm{obs}}(-h-k-l)$ are absent. Consequently there is no specific influence operating on the model intensities $I_{\text {model }}(h k l)$ and $I_{\text {model }}(-h-k-l)$ in the least-squares refinement forcing them to become equal by way of a Flack parameter close to 0.5. As a result the Flack parameter is not driven to any particular value and will tend to stay close to or 'stick at' its starting value. Other details of this argument are given by Flack \& Bernardinelli (2006). All but one of the structure analyses contributing to Table 2 used software that uses a starting value of 0.0 for the Flack parameter. The single exception is the value of 0.57 for which the starting value of the Flack parameter was 0.5. It is our contention that, were the crystal structures contributing to Table 2 refined in the noncentrosymmetric model but with data corresponding to a Friedel coverage of $100 \%$, then values of the Flack parameter close to 0.5 would be obtained.

The topic of a suitable or necessary data-measurement region was treated early on by Bernardinelli \& Flack (1987) in a study using enantiopure potassium hydrogen $(2 R, 3 R)$ tartrate. It was found that refinement of the Flack parameter was stable and unbiased when using a Friedel coverage of either $100 \%$ or $0 \%$, in the latter case with several different choices of data region, e.g. $h \mathrm{k} l$ all positive or all negative. The standard uncertainty of the Flack parameter obtained with a Friedel coverage of $0 \%$ was higher than when using a coverage of $100 \%$ due to the diminished number of observations. The results of the Bernardinelli \& Flack (1987) and the present study are not in contradiction. The early study concerned a crystal structure which was undoubtedly non-centrosymmetric with significant resonant scattering. The current study applies to centrosymmetric structures mistakenly analysed as being non-centrosymmetric.

All in all, of the 231 centrosymmetric structure analyses treated as non-centrosymmetric, $86 \%$ of them $(=198 / 231$; $198=231-28-5)$ are to be considered as unsatisfactory from the point of view of measurement, data reduction, refinement or reporting. Of course, $100 \%$ of these structure solutions are unsatisfactory due to an incorrect choice of space group.

It must be stressed that the real crystal structures contributing to Tables 1 and 2 are centrosymmetric. Consequently, the Flack parameter is undefined and has no physical meaning for them as a description of absolute structure. One perceives in this situation that the value obtained for the Flack parameter of the non-centrosymmetric model is a junk parameter as concerns an absolute-structure specification of the real crystal structure. Its refined value is dictated by other incidental factors as described in this section. In accordance with the view of Wilson (1975), 'Do the right calculation and get the right answer... all that really needs to be said is' that the authors of these papers should have had available a Friedel coverage of $100 \%$ in the discovery or structure-determination phase of the analysis and should have realised for themselves that the crystal structure was centrosymmetric.

\section{Pseudo-centrosymmetric structures}

There are no published lists of pseudo-centrosymmetric structures. Nevertheless, those authors who study the literature to detect crystal structures which are faulty in one way or another all have their own unpublished material for structures which appeared erroneous at first glance but on further examination turned out to be correct. This material includes those structures which are nearly centrosymmetric but are definitely non-centrosymmetric. A table containing the pertinent aspects of the set of 180 crystal-structure analyses is presented in the supplementary material. Many, but not all, of these structures are deemed to be non-centrosymmetric because they contain an enantiopure molecule or group. The data selection and extraction procedures were very similar to those used for the centrosymmetric structures described in $\$ 2$. We will just mention the differences here. The only selection criterion was that the paper had been published in or after 1993, a relaxation of the previous 1995 limit which was too restrictive. An additional keyword disorder was used on the searchable electronic documents. In a few instances there were direct contacts with the principal author of the paper. Such instances are marked in the notes to the compound. The measure of the pseudo-centrosymmetry of each structure was obtained from PLATON (Spek, 2003). In fact, PLATON produces one fit parameter for each symmetry operation to be added. Often, but not always, the values of these fit parameters are identical. When this is not the case, we adopt the practice of reporting the lowest value. A measure of the amount of resonant scattering for each data set was obtained by use of the formula (Girard et al., 2003)

$$
\text { Rescat }=10^{4}\left[2\left(\Sigma f^{\prime \prime 2}\right) /\left(\Sigma f^{2}\right)\right]^{1 / 2} .
$$

Of the 180 crystal structures, 78 had insufficient treatment and reporting of absolute-structure determination; for eight we were unsure whether the structures are centrosymmetric or non-centrosymmetric; another 14 were judged to be definitely centrosymmetric and included in the analysis in $\$ 2$; and 48 had absolute-structure information but had been measured with zero or low Friedel coverage. The final set of 32 structures had absolute-structure information and had been measured with a Friedel coverage of $100 \%$. So $82 \%$ of these structure determinations and reports were deficient in one way or another. In view of the results of $\S 2$, it is only on this final set of structures measured with a high Friedel coverage that we have concentrated our attention.

Table 3 contains the most important data harvested from the pseudo-centrosymmetric structures measured with a Friedel coverage of $100 \%$. It is immediately apparent from 
Table 3

Pseudo-centrosymmetric crystal structures refined with a Friedel coverage of $100 \%$.

Fit, Rescat and Flack $x$ are defined in the text. The table is arranged columnwise in ascending order of Rescat, the degree of resonant scattering in the data.

\begin{tabular}{rrc}
\hline Fit & Rescat & Flack $x$ \\
\hline 83 & 4 & $-0.5(16)$ \\
95 & 6 & $-3(2)$ \\
83 & 7 & $1.0(10)$ \\
92 & 8 & $-1.9(6)$ \\
94 & 8 & $1(2)$ \\
88 & 8 & $0.1(6)$ \\
92 & 25 & $-0.1(2)$ \\
90 & 34 & $0.2(2)$ \\
87 & 36 & $0.2(4)$ \\
92 & 36 & $0.03(15)$ \\
88 & 38 & $0.21(9)$ \\
80 & 39 & $0.1(3)$ \\
89 & 115 & $0.06(3)$ \\
88 & 116 & $0.50(10)$ \\
96 & 127 & $-0.01(3)$ \\
93 & 141 & $0.27(7)$ \\
95 & 167 & $-0.06(9)$ \\
100 & 183 & $-0.021(18)$ \\
97 & 229 & $0.05(4)$ \\
90 & 235 & $-0.012(13)$ \\
96 & 256 & $0.00(4)$ \\
93 & 273 & $0.000(9)$ \\
93 & 273 & $-0.014(13)$ \\
82 & 315 & $-0.001(8)$ \\
80 & 338 & $-0.04(2)$ \\
87 & 360 & $-0.012(14)$ \\
93 & 395 & $0.03(3)$ \\
96 & 608 & $0.04(2)$ \\
100 & 696 & $-0.009(11)$ \\
91 & 1039 & $0.008(5)$ \\
100 & 1320 & $0.017(10)$ \\
100 & 1391 & $-0.006(12)$ \\
\hline & &
\end{tabular}

Table 3 that with a significant resonant-scattering contribution (medium to large value of Rescat) and a Friedel coverage of $100 \%$ it is frequently possible to obtain an absolute-structure determination by way of the Flack parameter despite the structure being very nearly centrosymmetric as witnessed by the PLATON fit parameter. It is also very clear that as the amount of resonant scattering increases, so the standard uncertainty on the Flack parameter diminishes. At low values of Rescat, large standard uncertainties and large deviations from zero are observed for the Flack parameter. For compounds with Rescat $>160$ (see $\S 4$ ) the average value of the Flack parameter of -0.0019 is close to the expected value of zero. For detailed information on the two compounds [DOJYAC, $x=0.50$ (10), and MAKDAE, $x=0.27$ (7)] for which Rescat $>80$ and the Flack parameter is significantly different from zero, the notes in the supplementary publication should be consulted. Counter to our own intuition and to that of others (Jones, 1984; Iwasaki, 1974; Cianci et al., 2005), the Flack parameter works well in situations where the crystal structure is definitely non-centrosymmetric but strongly pseudo-centrosymmetric. Why?

A straightforward analysis will enable us to understand why reliable values of the Flack parameter may be obtained from pseudo-centrosymmetric crystal structures. A suitable model consists of a structure with two components:

(i) (C), a centrosymmetric part formed of resonant atoms giving a contribution, $\mathbf{F}_{\mathrm{c}}(\mathbf{h})$, to the complete structure factor, $\mathbf{F}(\mathbf{h})$, which may be written as $\mathbf{F}_{\mathrm{c}}(\mathbf{h})=s\left(C+i C^{\prime \prime}\right)$, where $s=+1$ or -1 according to the sign of the partial structure factor $\mathbf{F}_{\mathrm{c}}(\mathbf{h})$, and $C$ and $C^{\prime \prime}$ are its non-resonant and resonant atomic scattering amplitudes, $\mathbf{F}_{\mathrm{c}}(\mathbf{h})$ being obtained by a sum over all atoms in the unit cell. $\mathbf{F}_{\mathrm{c}}(\mathbf{h})$ may be written in this form even if the atoms in the unit cell are of various chemical elements.

(ii) (N), a non-centrosymmetric part formed of resonant atoms all of the same chemical element. This part gives a contribution to $\mathbf{F}(\mathbf{h})$ of $\mathbf{F}_{\mathrm{n}}(\mathbf{h})$ which may be written as

$$
\mathbf{F}_{\mathrm{n}}(\mathbf{h})=\left(N+i N^{\prime \prime}\right)\left(\cos \varphi_{\mathrm{n}}+i \sin \varphi_{\mathrm{n}}\right)
$$

where $N$ and $N^{\prime \prime}$ are the non-resonant and resonant atomic scattering amplitudes and $\varphi_{\mathrm{n}}$ is the phase angle of the structure factor, $\mathbf{F}_{\mathrm{n}}(\mathbf{h})$ being obtained by a sum over all atoms in the unit cell.

The total structure factor $\mathbf{F}(\mathbf{h})=\mathbf{F}_{\mathrm{c}}(\mathbf{h})+\mathbf{F}_{\mathrm{n}}(\mathbf{h})$ and $\mathbf{F}(-\mathbf{h})$ may be expressed in terms of the same quantities by reversing the $\operatorname{sign}$ of $\sin \varphi_{\mathrm{n}}$. In a crystal twinned by inversion the intensities of the reflection $\mathbf{h}$ and its Friedel opposite $-\mathbf{h}$ are $I(\mathbf{h})=(1-x)|\mathbf{F}(\mathbf{h})|^{2}+x|\mathbf{F}(-\mathbf{h})|^{2}$ and $I(-\mathbf{h})=x|\mathbf{F}(\mathbf{h})|^{2}+(1-$ $x)|\mathbf{F}(-\mathbf{h})|^{2}$. The critical quantity $\Delta(\mathbf{h})$ is the difference in intensity between reflections $\mathbf{h}$ and $-\mathbf{h}$,

$$
\begin{aligned}
\Delta(\mathbf{h}) & =I(\mathbf{h})-I(-\mathbf{h})=4(1-2 x) s \sin \varphi_{\mathrm{n}}\left(C^{\prime \prime} N-C N^{\prime \prime}\right), \\
|\Delta(\mathbf{h})| & =4|1-2 x|\left|\sin \varphi_{\mathrm{n}}\right|\left|C^{\prime \prime} N-C N^{\prime \prime}\right| .
\end{aligned}
$$

The latter equation tells us much of what we need to know. For a Friedel pair of reflections to have a large absolute difference it is best for $\varphi_{\mathrm{n}}$ to be near $90^{\circ}$ or $270^{\circ}$. An Argand diagram of the complex plane indicating $\mathbf{F}_{\mathrm{c}}(\mathbf{h}), \mathbf{F}_{\mathrm{n}}(\mathbf{h})$ and $\mathbf{F}_{\mathrm{n}}(-\mathbf{h})$ confirms this result. One way to make the term $\left|C^{\prime \prime} N-C N^{\prime \prime}\right|$ large is to set $C^{\prime \prime}$ large and $N^{\prime \prime}$ to zero. Indeed this is probably the case for many of the pseudo-centrosymmetric structures studied here. These structures consist of an inner centrosymmetric part often with a heavy metal atom of large $C^{\prime \prime}$ at the centre and an outer non-centrosymmetric part of light atoms with $N^{\prime \prime}$ $\simeq 0$. These conditions systematically maximize $|\Delta(\mathbf{h})|$. One should note especially that for the best contrast $|\Delta(\mathbf{h})|$, curiously the resonant atoms are in a centrosymmetric arrangement and the non-centrosymmetric part of the structure (possibly chiral) is composed of non-resonant atoms.

\section{Predicting the uncertainty of the Flack parameter}

Table 3 presented the results on pseudo-centrosymmetric crystal structures reporting values of the Flack parameter determined with a high Friedel coverage. We already pointed out in $\$ 3$ that as the amount of resonant scattering (Rescat) increases so the standard uncertainty $(u)$ on the Flack parameter diminishes. It is possible to quantify this observation to some extent. We restrict our attention to data with high Friedel coverage for this is the sure way to undertake absolute-structure determination. In Fig. $1, \log _{10}(u)$ is plotted 


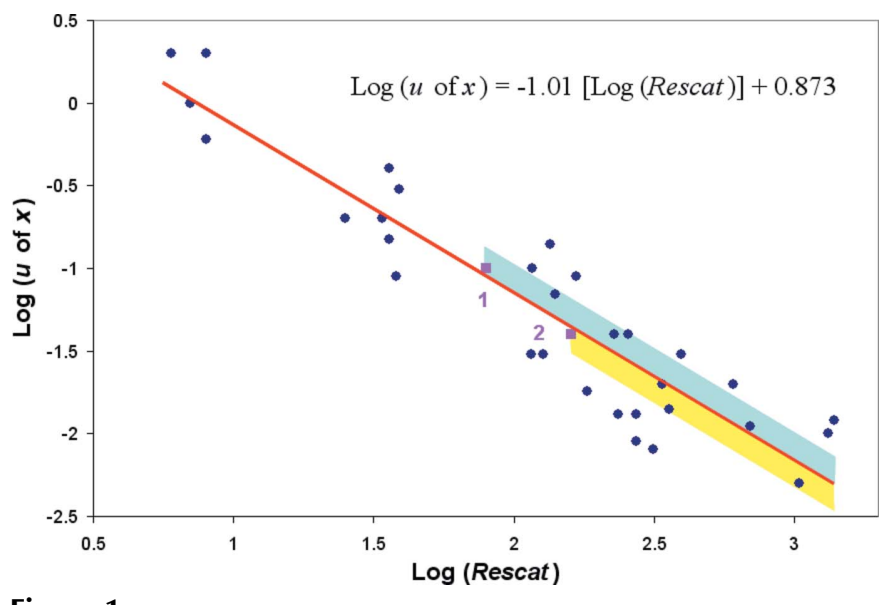

Figure 1

$\log (u)$ versus Rescat for 28 pseudocentrosymmetric crystal structures measured with a Friedel coverage of $100 \%$. Point 1: $u=0.1$, Rescat $=80$ with the upper (darker) band for enantiopure compounds; point 2: $u=$ 0.04 , Rescat $=160$ with the lower (lighter) band for the general case, are useful for prediction and evaluation.

against $\log _{10}$ (Rescat) for the values in Table 3. Although there is a fair amount of scatter of the points in this plot, the general tendency shows a linear variation of $\log (u)$ with $\log ($ Rescat) and may be represented by a best straight line: $\log _{10}(u)=$ $-1.01 \log _{10}$ (Rescat) +0.873 . On this line, a value of $u=0.04$ corresponds to a value of Rescat $=160$, and a value of $u=0.10$ corresponds to a value of Rescat $=80$. These two values of $u$ are the limiting values chosen by Flack \& Bernardinelli (2000) as upper limits in the general case $(u=0.04)$ and with a compound known to be enantiopure $(u=0.10)$ for absolutestructure determination. Consequently, the corresponding values of Rescat (160, general, and 80, enantiopure) are lower limits for absolute-structure determination calculable from a knowledge of the elemental composition of the compound alone. These two values are thus of practical use in the choice of compound and radiation wavelength for absolute-configuration determination prior to experimentation and in the evaluation of the value of $u$ obtained. Of course, Fig. 1 has been derived from the pseudo-centrosymmetric structures that we had available and it is difficult to know how the relation between $\log (u)$ and $\log ($ Rescat $)$ might look for a more general class of compounds measured with a Friedel coverage of $100 \%$.

\section{Centrosymmetric structures giving a high standard uncertainty on $x$}

For the five centrosymmetric crystal structures in $\$ 2$ which gave large standard uncertainties on the Flack parameter, we have examined various causes for this effect. The following effects are not responsible for the high uncertainty of the Flack parameter in these structure determinations:

(i) Inadequate resonant scattering contribution. The parameter Rescat for these compounds takes values between 143 and 297 which is clearly adequate. (ii) Overall bad fit of the model. The conventional $R$ values lie between 0.047 and 0.085 so, although these structure determinations are not of the highest quality, the overall fit is adequate.

(iii) Inadequate data. The Friedel coverage is $100 \%$ in all five cases.

It is possible, however, to explain the high standard uncertainties on $x$ by supposing that the refined non-centrosymmetric model (remember the crystal structure itself is centrosymmetric) is very close to being centrosymmetric. Indeed the PLATON fit parameter for four of these structures is $100 \%$, the remaining structure having a value of $97 \%$. Expressing the scattering density in terms of a centrosymmetric $\left(C, C^{\prime \prime}\right)$ and an antisymmetric $\left(A, A^{\prime \prime}\right)$ component, we may use $\Delta(\mathbf{h})=4(1-2 x)\left(C^{\prime \prime} A-C A^{\prime \prime}\right)$, similar to the expression obtained in $\S 3$. The approach to a centrosymmetric arrangement is characterized by the values of $A$ and $A^{\prime \prime}$ vanishing simultaneously, resulting in tiny values of $|\Delta(\mathbf{h})|$ and $\partial \Delta(\mathbf{h}) / \partial x$, and consequently large values of the standard uncertainty of the Flack parameter. A high value of the standard uncertainty of the Flack parameter for a reasonable value of Rescat may be used as another sign that the crystal structure is centrosymmetric.

\section{Bias of the Flack parameter}

Preliminary statistical tests on crystal structures determined by three separate groups of structure analysts in Geneva (Bernardinelli), Utrecht (Spek) and Zurich (Linden) seem to indicate that there may be a small systematic negative bias in the values of the Flack parameter. The average values of $x$ and the number of structures used in the survey are as follows: Geneva, -0.0050 for 109 structures; Utrecht, -0.0097 for 160 structures; Zurich, -0.014 for 80 structures. Although the bias is small, its significance seems to be confirmed by the fact that the three groups of structure analysts have different equipment, operate at different wavelengths, use various software and follow their own procedures. Moreover the choice of compounds studied is varied and independent.

A brain-storming session over the possible cause(s) of such a bias brought forward many suggestions which we now list. Certain apparatus might overestimate or underestimate strong or weak intensities. Weak reflections may be recorded with too-high an intensity owing to a contribution from multiple reflection. Background estimation may be systematically in error. $f^{\prime \prime}$ values may be systematically biased. Weighting schemes may play a role. Overestimated atomic displacement parameters due to the lack of an extinction or absorption correction would lead to a negative bias of the Flack parameter. No definite statement can be made at this stage.

We recall that Tables 1 and 2 present values of the Flack parameter obtained from centrosymmetric crystals refined with a non-centrosymmetric model. It is not appropriate to talk of or analyse bias for such data as the expected value of the Flack parameter of a centrosymmetric crystal is undefined. Tables 1 and 2 contain values of a junk parameter of the real crystal structure. 


\section{Inconsistent chemical and crystallographic data}

Crystal-structure analyses arise in which the crystallographic and chemical evidence are in conflict. Over 20 cases have been identified by Djukic et al. (2006) in a review of planar-chiral metallacycles. Often the compound has been declared to be enantiopure but the structure analysis results in a Flack parameter significantly close to 0.5 or even in the crystal structure of a racemate. The twinning-by-inversion model of the Flack parameter implies, for a value close to 0.5 , that the compound is a racemate, in contradiction with the chemical evidence or hypothesis. We shall mention a few effects which may be at the origin of inconsistencies of this type:

(i) Although Friedel opposites are measured, their intensities are averaged before structure refinement.

(ii) A semi-empirical absorption correction has been applied which attempts to reduce intensity differences between Friedel opposites.

(iii) An unconverged least-squares refinement has been undertaken due to inappropriate procedures.

(iv) The bulk compound is a racemate (in contradiction to the chemical evidence presented) and crystallizes by spontaneous resolution but giving crystals that are twinned by inversion.

(v) The enantiopurity of the bulk compound is high but not exactly $100 \%$. The crystal chosen for the diffraction experiment may then be that of the racemate. Cases where the temperature of fusion of the crystalline racemate is much higher than that of the enantiopure compound lead to phase diagrams in which the order of the $e e$ 's is: enantiopure $(100 \%)$ $>$ eutetic $>$ bulk $>$ racemate $(0 \%)$, the eutetic being closer to enantiopurity than the bulk. In such cases the first crystals to appear are those of the racemate.

In general, resolving such ambiguities requires more information than is available in the publication. In such cases characterization of the bulk and the individual crystal used for the diffraction experiment is crucial. The most suitable measurements are those of CD spectra in solution or solid, and enantioselective chromatography. On the diffraction side it is advisable to check the diffraction apparatus and all related software by measurement of a well characterized standard enantiopure compound containing significant resonant scattering.

\section{Concluding remarks}

Cianci et al. (2005) have recently published a very detailed review of the application of resonant scattering to problems in structural chemistry and biology. This text misses no opportunity to highlight the considerable number of past achievements and future potential of synchrotron radiation to structure analysis. However, Cianci et al. (2005) make no mention of how future developments in synchrotron-radiation technology and measurement techniques could be of help in the common problem of the determination of absolute configuration from light-atom small-molecule crystal structures. Doubtless, Cianci et al. (2005) have in mind that the $K$ absorption edges of light atoms occur at long wavelengths, making diffraction data very sparse, owing to a small Ewald sphere, and experimentation difficult, owing to high absorption (see e.g. Biou et al., 2005).

It is very clear indeed from the results presented in $\S 2$ and $\S 3$ that provided the value of Rescat is larger than a threshold value of perhaps 40 , it must be considered mandatory to measure and use a Friedel coverage of $100 \%$ for a new crystal structure presented as being non-centrosymmetric. If this is done, for centrosymmetric structures, values of the Flack parameter close to zero due to 'sticking' will be avoided and values close to 0.5 will be obtained. Thus, as long as a Friedel coverage of $100 \%$ has been used, a value of the Flack parameter significantly close to 0.5 may be taken as one indication that the crystal structure may in fact be centrosymmetric, and a value significantly close to zero that it is definitely noncentrosymmetric. It is not valid to justify the choice of a noncentrosymmetric space group by a value of the Flack parameter close to zero if the Friedel coverage is not close to $100 \%$. It must be borne in mind that a value of the Flack parameter significantly close to 0.5 for a truly non-centrosymmetric structure indicates that the macroscopic crystal is twinned by inversion in a ratio of approximately 50:50. In the late stages of analysis, we thoroughly recommend the evaluation of the crystal structure by procedures such as PLATON in order to detect missed symmetry.

We recall yet again that, for estimates of the Flack parameter and its standard uncertainty to be valid, they need to be determined by a converged full-matrix least-squares refinement. In this respect the advice of Clegg (2003) is of the very greatest importance.

We hope that many engaged in the endeavour of crystalstructure determination, evaluation, reporting and archiving will have enjoyed, or at least benefited from, reading this paper. Our study confirms that crystal structures are far too often described with incorrect space groups or that crucial information is missing from a paper and its associated supplementary publications. Thus, standards of authorship, refereeing, software writing, automated evaluation, editing and publication need to be further improved for all the journals that we have encountered. Although Acta Crystallographica Section B, Section $C$ and Section $E$ achieve a very high quality in their published material, one suspects from the following remark of Clegg \& Watson (2006) that this is often not the case for submitted manuscripts! 'Papers which are difficult to understand, which fall well short of the requirements of the Notes for Authors, or which require extensive text correction and editing will be returned to the author for revision without a detailed review; it is the responsibility of authors, not of Co-editors, to generate an acceptable text for the Abstract, Comment and Experimental sections and to ensure that other CIF items are correct and complete.'

Our heartfelt thanks go to Dr R. E. Marsh for having supplied us with his list of pseudo-centrosymmetric structures which formed the basis of a very considerable part of this 
work. He certainly merits to be included as an author of this paper but declined this due recognition, feeling he had not contributed sufficiently. We appreciated the excellent service provided by the Cambridge Crystallographic Data Centre in making available supplementary material from their CIF archive.

\section{References}

Bernardinelli, G. \& Flack, H. D. (1987). Acta Cryst. A43, 7578.

Biou, V., Bösecke, P., Bois, J.-M., Brandolin, G., Kahn, R., Mas, C., Nauton, L., Nury, H., Pebay-Peyroula, E., Vicat, J. \& Stuhrmann, H. (2005). J. Synchrotron Rad. 12, 402-409.

Cianci, M., Helliwell, J. R., Helliwell, M., Kaucic, V., Logar, N. Z., Mali, G. \& Tusar, N. N. (2005). Crystallogr. Rev. 11, 245-335.

Clegg, W. (2003). Acta Cryst. E59, e2-e5.

Clegg, W. \& Watson, D. G. (2006). Acta Cryst. E62, e8-e9.

Clemente, D. A. (2005). Inorg. Chim. Acta, 358, 1725-1748.

Clemente, D. A. (2006). To be published.

Clemente, D. A. \& Marzotto, A. (2003). Acta Cryst. B59, 4350 .

Clemente, D. A. \& Marzotto, A. (2004). Acta Cryst. B60, 287292.
Djukic, J.-P., Hijazi, A., Flack, H. D. et al. (2006). Chem. Soc. Rev. In preparation.

Flack, H. D. (1983). Acta Cryst. A39, 876-881.

Flack, H. D. \& Bernardinelli, G. (2000). J. Appl. Cryst. 33, 11431148.

Flack, H. D. \& Bernardinelli, G. (2006). Inorg. Chim. Acta, 359, 383 387.

Girard, E., Stelter, M., Vicat, J. \& Kahn, R. (2003). Acta Cryst. D59, 1914-1922.

Herbstein, F. H., Hu, S. \& Kapon, M. (2002). Acta Cryst. B58, 884 892.

Herbstein, F. H. \& Marsh, R. E. (1998). Acta Cryst. B54, 677-686.

Iwasaki, H. (1974). Acta Cryst. A30, 173-176.

Jones, P. G. (1984). Acta Cryst. A40, 660-662.

Marsh, R. E. (1999). Acta Cryst. B55, 931-936.

Marsh, R. E. (2002). Acta Cryst. B58, 893-899.

Marsh, R. E. (2004). Acta Cryst. B60, 252-253.

Marsh, R. E. (2005). Acta Cryst. B61, 359.

Marsh, R. E., Kapon, M., Hu, S. \& Herbstein, F. H. (2002). Acta Cryst. B58, 62-77.

Marsh, R. E. \& Spek, A. L. (2001). Acta Cryst. B57, 800-805.

Spek, A. L. (2003). J. Appl. Cryst. 36, 7-13.

Wilson, A. J. C. (1975). In Anomalous Scattering, edited by S. Ramaseshan \& S. C. Abrahams, pp. 325-331. Copenhagen: IUCr/ Munksgaard. 